\title{
Editorial: Manipulating Nutrient Intake Circadian Patterns: An Economical Therapeutic Strategy
}

\author{
Akbar Nikkhah* \\ Chief Highly Distinguished Professor, Department of Animal Sciences, Faculty of Agricultural Sciences, \\ University of Zanjan, Zanjan 313-45195 Iran
}

This article establishes a circadian chronological basis for food intake orchestration in modern and postmodern humans, and illuminates roadmaps for future real-world research.

Chronophysiology is an evolutionary multiscience that enables animals including humans to cope with the fluctuating environment. Timing of food intake has been discovered to orchestrate postprandial circadian rhythms of nutrient ingestion, digestion, transport, and metabolism. As such, chrono-orchestration of food intake regulates appetite and eating rate. Chrononutritional physiology is a major unnoticed healthy science that, in light of realistic modeling and understanding of voluntary feed intake in foodproducing ruminant models, offers practical perceptions towards establishing health-improving feasible nutritional programs and regimens [1-5]. This is crucial considering that reliable hunger and nutrient intake predictions are indispensable to healthy and onchophobic provision of foods and nutrients to human cells. Such insightful knowledge can help formulate guidelines to prevent overnutrition and health issues namely overweight gain, obesity, and diabetes [4].

The evolutionary patterns of food searching and intake behavior have contributed to circannual and circadian patterns in endocrinology and metabolism. The severe modifications in life style due to transition from tradition towards modernity and postmodernity, natural rhythms of appetite and nutrient intake have changed. As a result of less natural eating and overnutrition particularly in fats and sugars overnutrition, it has become difficult to maximize synchronies between external environments and internal physiological states. Untimely food intake has exacerbated the modern problems by increasing risks of obesity, glucose intolerance, insulin resistance,

*Address correspondence to this author at the Chief Highly Distinguished Professor Department of Animal Sciences, Faculty of Agricultural Sciences, University of Zanjan, Zanjan 313-45195 Iran; Tel: +98-24-35052801;

Fax: +98-24-35053202; E-mail: anikkha@yahoo.com, nikkhah@znu.ac.ir diabetes mellitus, and resulting cardiovascular irregularities [3].

Timing of food intake is a major feasible life manager that significantly affects how quickly nutrients are ingested, how extensively they are assimilated, and how proportionately the resulting substrates are distributed among different functions (i.e., deposition, oxidation, secretion, and excretion). These mechanisms are real-life scientific tools whereby the modern man will be able to optimize life quality and satisfaction, health indices, and longevity. However, circadian food intake chronology has not yet been accommodated in dietary reference intake guidelines [6].

Therefore, chronobiological management practices (e.g., circadian food intake timing and frequency) offer viable and pragmatic models to improve nutrient transformation and utilization. These therapeutic strategies can well be practiced by all, particularly overweight people, diabetics, shift-workers, and athletes preparing for professional matches and games. Future research will need to explore data on nutrigenomics and immunopathology of food intake chronology. This is a path wherein therapeutic nutritional chronophysiology will offer innovative strategies towards quality life.

Thankfully acknowledged are The Ministry of Science, Research and Technology and University of Zanjan, Iran, for supporting the author's programs of optimizing the new millennium global science education.

\section{REFERENCES}

[1] Nikkhah A. Chronophysiology of ruminant feeding behavior and metabolism: an evolutionary review. Biol Rhythm Res 2013; 44(2): 197-218. http://dx.doi.org/10.1080/09291016.2012.656437

[2] Nikkhah A. Bioscience of ruminant intake evolution: feeding time models. Adv Biosci Biotechnol 2011a; 2: 271-274. http://dx.doi.org/10.4236/abb.2011.24039 
[3] Nikkhah A. Ruminant chronophysiological management: an emerging bioscience. Open Access Anim Physiol 2011b; 3: 9-12. http://dx.doi.org/10.2147/OAAP.S24071

[4]

Nikkhah A. Science of eating time: A novel chronophysiological approach to optimize glucose-insulin dynamics and health. J Diab Mellit 2011c; 2(1): 8-11. http://dx.doi.org/10.4236/jdm.2012.21002
[5] Nikkhah A. Timing of feed presentation entrains periprandial rhythms of energy metabolism indicators in once-daily fed lactating cows. Biol Rhythm Res 2012; 43(6): 651-661. http://dx.doi.org/10.1080/09291016.2011.631773

[6] Dietary Reference Intakes (DRI). Recommended Intakes for Individuals, Food and Nutrition Board, Institute of Medicine, National Academies. National Academy of Sciences, Washington, D.C. USA 2007. 